\title{
GRANDES PROCESOS DE CAMBIO Y SUS IMPACTOS SOBRE LA DISCAPACIDAD INTELECTUAL
}

\author{
María Isabel García Rodríguez \\ Instituto de Estudios Sociales Avanzados. Consejo Superior de Investigaciones \\ Científicas (España)
}

\begin{abstract}
Resumen. Este artículo teórico trata de revisar los grandes procesos de cambio producidos en las últimas décadas y los impactos sobre el tratamiento de la discapacidad intelectual. El objetivo es revistar el significado que han tenido la globalización y la construcción de la Unión Europea sobre el Estado del bienestar y, en particular, sobre el tratamiento de la discapacidad intelectual, teniendo en cuenta cómo se aborda en la legislación internacional. Para ello se ha realizado una revisión bibliográfica que analiza dichos cambios y la legislación internacional que recoge el tratamiento de la discapacidad intelectual en los próximos años. Las principales conclusiones a las que nos conduce esta revisión son que los grandes cambios políticos y sociales han conducido a la redefinición del Modelo Social Europeo en términos de reducción del gasto público, lo cual nos plantea dudas sobre la posibilidad de implementar las políticas sociales sobre discapacidad intelectual tal y como se recogen en la legislación internacional. Estos hechos plantean dudas también acerca de la posibilidad de implementar políticas sociales sobre la discapacidad intelectual según lo establecido en el derecho internacional.

Palabras clave: Discapacidad intelectual, Estado del bienestar, Modelo Social Europeo.
\end{abstract}

\section{GREAT CHANGE PROCESSES AND ITS IMPACTS ON INTELLECTUAL DISABILITY}

\begin{abstract}
This theoretical article tries to review the great processes of change produced in the last decades and their impacts on the treatment of intellectual disability. The main objective is to examine, from a theoretical point of view, the significance of globalization process and the impact of European Union construction on the welfare states. We are interested particularly on the treatment of intellectual disability, taking into account how it is dealt with in international law. For this purpose, a bibliographic review has been carried out, analyzing these changes and the international legislation that addresses the treatment of intellectual disability in the coming years. This review has lead us to a main conclusion, namely, great political and social changes have defined the European Social Model again in terms of reducing social budgets for social policies. These facts raise doubts about the possibility of implementing social policies on intellectual disability as set out in international law.
\end{abstract}

Keywords: Intellectual Disability, Welfare State, European Social Model.

\section{PROCESSOS DE MUDANÇA GRANDES E SEU IMPACTO SOBRE DEFICIÊNCIA INTELECTUAL}

Resumo. Este artigo teórico tenta rever os principais processos de mudança produzida nas últimas décadas e os impactos sobre o tratamento da deficiência intelectual. O objetivo é revistar a partir do ponto de vista teórico, o significado que tiveram a globalização ea União Europeia sobre o estado de bem-estar e, em particular, sobre o tratamento da deficiência intelectual, considerando como abordado no direito internacional. Com essa intenção izemos uma revisão da literatura que examina essas mudanças e 
legislação internacional abordando o tratamento da deficiência intelectual nos próximos anos. As principais conclusões que leva este comentário é que as grandes mudanças políticas e sociais levaram à redefinição do Modelo Social Europeu em termos de redução da despesa pública, o que levanta dúvidas sobre a possibilidade de implementação de políticas sociais sobre deficiência intelectual como refletido no direito internacional.

Palavras chave: Deficiência intelectual, Estado do bem-estar, Modelo Social Europeu

\section{Introducción}

En al artículo se realiza un análisis sobre el impacto que tienen procesos trasnacionales como la globalización y la construcción de la Unión Europea sobre la cohesión social. Para ello observaremos los cambios que se han producido en el Modelo Social y sobre los Estados del bienestar por ser estos los instrumentos que la hacen efectiva. Asimismo, en el artículo se analiza la situación de las personas con discapacidad en la legislación internacional, que se concreta en los Estados mediante diferentes planes de acción. Con este punto de partida trataremos de dar cuenta de las posibilidades reales de que estas actuaciones se implementen de la manera esperada, dadas las reformas que se están llevando a cabo en los Estados de bienestar.

\section{Método}

Dado que se trata de un ensayo sobre el futuro de la cohesión social en España y la influencia que tiene sobre ella el marco internacional, el artículo tiene un contenido fundamentalmente teórico. El método llevado a cabo, por tanto, ha sido la revisión bibliográfica relacionada con aquellos asuntos que se tratan de manera principal, como son: el Estado del bienestar y su evolución y el surgimiento de nuevos riesgos sociales como consecuencia de los cambios políticos, económicos y sociales. También se ha revisado la normativa internacional, la norma española que recoge la atención a las personas en situación de dependencia, y los planes de acción aprobados recientemente, que tienen como población diana a personas con discapacidad intelectual.

La búsqueda bibliográfica se ha llevado a cabo a través de las bases de datos: Elsevier, JSTOR y Dialnet, a las cuales se ha accedido a través de la Biblioteca virtual del CSIC.

Los descriptores utilizados han sido: Estado del bienestar, Modelo Social, Globalización, Nuevos Riesgos Sociales, Dependencia, Planes de inclusión Discapacidad, Discapacidad intelectual, Europa Social.

\section{Resultados}

Hace cuatro décadas España comenzó su andadura democrática tratando de aproximarse a las sociedades europeas avanzadas, cuya estructura política y social iba a corresponder con la de los Estados Democráticos de Derecho, que disfrutaban de niveles de protección social altos, proporcionados por sus respectivos Estados de bienestar. España comenzó la construcción de sus estructuras de bienestar cuando en Europa ya se estaban planteando debates sobre la sostenibilidad de aquéllas, en un 
contexto afectado por las consecuencias económicas de la crisis de los años 70 y por los cambios en los sistemas económicos productivos. Esta crisis supuso un primer impacto negativo sobre los Estados de bienestar, que los condujo a encajar una serie de restricciones o cambios que conformaron lo que se ha venido a llamar el paso de la "Edad de Oro" a la "Edad de Plata" de los Estados de bienestar (Moreno, 2009).

Lo cierto es que en los años ochenta se produjo un punto de inflexión. Los discursos políticos y las medidas aplicadas por Reagan y Thatcher en sus respectivos países (Del Pino, 2004) aportaron el impulso que finalmente dio lugar a un conjunto sustantivo de reformas sobre los Estados de bienestar ${ }^{1 .}$

Aquellos discursos y medidas estuvieron mediatizados por el desarrollo de dos procesos trasnacionales de extraordinaria importancia como son: la globalización y la construcción de la Unión Europea, ambos vinculados. Su desarrollo ha enfrentado a los Estados a complejos procesos de cambio, internos y externos, en todos los ámbitos: económico, social y político. En los espacios nacionales, los Estados pusieron en marcha el proceso para hacer efectivas las exigencias propias de la globalización neoliberal y las reformas que requería la incorporación a la Unión, mediante la firma de Tratados y Pactos que vinculaban su futuro al desarrollo de estos procesos que, aún hoy, está lejos de concluir. Estos compromisos significan la renuncia de los Estados a parte de su autonomía, por ejemplo, en política monetaria, aunque otros ámbitos de competencia como el social, siguen vinculados al ámbito nacional. Esta situación mantiene la desigualdad porque no se introducen medidas comunes para mantener la cohesión social.

\section{El proceso de globalización}

Son muchos los autores que han formulado teorías sobre la globalización, con enfoques muy diversos y también más o menos complejos, según el aspecto en el que se centren. Sin embargo, parece existir un consenso académico entre los autores respecto a su origen y sus consecuencias. Uno de los aspectos sobre los que existe mayor acuerdo es que el mundo está organizado según las necesidades del sistema económico capitalista, que su organización está erosionando la hegemonía de los Estados -Nación al tiempo que se incrementa la de corporaciones y organizaciones trasnacionales, lo cual tiene consecuencias graves para las economías y culturas locales y nacionales.

Lo relevante de la globalización es que se trata de un proceso muy complejo que tiene unos efectos directos sobre la vida de los individuos, aunque a primera vista pudiera no parecerlo. Tomaremos como ejemplo algunos de los elementos que han influido en los Estados desde hace unas décadas (Sholte, 2008), que son importantes porque se han traducido en los principios de regulación de las sociedades y de la vida de los ciudadanos.

- Uno de ellos es la internacionalización. Se trata del crecimiento de transacciones y de la interdependencia entre los países, de ahí que las actuaciones nacionales tengan límites claros, particularmente cuando los estados forman parte de tratados supranacionales.

- $\quad$ El otro es la liberalización, que lleva consigo la eliminación de las restricciones a los movimientos de recursos entre países. Respecto a esta cuestión, en la literatura se han planteado dos escenarios diferentes: por un lado, se prevé la configuración

\footnotetext{
${ }^{1}$ En los años noventa fueron algunos países Nórdicos los que reformaron sus Estados del bienestar, en dos mil fue Alemania, y recientemente, Holanda.
} 
de sociedades abiertas, más ricas y prósperas, y por otro, se prevé un incremento de la pobreza y del conflicto social, dadas las desigualdades que se crean entre unos países y otros.

Estos grandes procesos que afectan al funcionamiento de los Estados, no pueden ser considerados sin tener en cuenta a las mujeres, cuanto más si el objeto de análisis es la discapacidad, puesto que ellas son las principales proveedoras de cuidados y atención en los Estados de bienestar Mediterráneo, en los que persiste un grado de familización superior al de otros países que disfrutan de Estado de bienestar, como es el caso de los países nórdicos. La variable género debe ser introducida en el análisis, con el objetivo de conocer la diferencia que tienen los impactos de la globalización sobre las mujeres y sobre los hombres, sobre todo en lo que se refiere a su posición social. En este caso será útil la teoría política feminista porque identifica tres estructuras de autoridad a las que están sometidas las mujeres en el mundo: la estructura de clase (basada en el control de los medios de producción), la estructura de relación patriarcal (basada en la institución del matrimonio y de la maternidad) y la estructura de poder entre estado y política, que legitiman la reproducción del poder (Jónasdòttir, 1993). Esta formulación de las estructuras de poder, permite observar la globalización como un proceso problemático para las mujeres (Rubio, 2006), puesto que, lejos de minimizar dichas estructuras de poder, los cambios económicos que exige, las mantiene e incluso las refuerza.

Por otra parte, la globalización viene acompañada de un proceso de individualización que se manifiesta en diferentes niveles de la estructura social y en diferentes concepciones del Estado Social. Se trata de un término ambiguo que permite analizar la diferencia que existe entre los estilos de vida y el ejercicio de una verdadera autonomía (Simmel, 1990). En el caso que nos ocupa, el proceso debe ser analizado por su relación con el papel de la familia en la estructura social. En primer lugar, habrá que evaluar si el proceso de individualización es accesible para personas con discapacidad intelectual o si continúan vinculados a una situación de dependencia familiar. Si es así, en segundo lugar, habrá que determinar si el proceso de individualización permite a las mujeres abandonar sus funciones en la estructura familiar. La cuestión es que mientras la lógica social contemporánea exige a los individuos asumir la responsabilidad individual sobre sus vidas, la estructura familiar y buena parte de sus funciones se mantienen constantes. Así pues, las mujeres se ven sometidas a nuevas tensiones determinadas por la exigencia de seguir desempeñando los roles de género tradicionales y la necesidad de incorporarse a una estructura laboral que exige una posición social cada vez más individualizada (Beck 1998; Beck-Gersuing, 2003; Giddens, 1990; Meil, 2006).

Desde el punto de vista sociológico (Beck, 2003), la individualización está determinada por el grado de des-familización que alcanzan los Estados, a saber, por la capacidad que tienen los ciudadanos para desarrollar una vida autónoma, con unos determinados estándares de bienestar, sin requerir recursos a su familia, ya sean estos económicos o en forma de prestación de servicios. Para que esta situación sea posible es imprescindible la existencia de un Estado de bienestar que ponga en marcha políticas sociales de atención a la dependencia y a la autonomía personal, así como políticas que permitan la conciliación de la vida familiar y personal.

\section{El proceso de construcción europea}

La construcción europea como proceso complejo y multidimensional puede ser analizado desde diferentes perspectivas, sin embargo, nuestra preocupación aquí es la 
evolución del Modelo Social Europeo, dada su relación con la cohesión social y los impactos que produce sobre los ciudadanos más vulnerables.

Aunque la construcción de la Unión de Europea es un proyecto hegemónico, no está exento de contradicciones. Una de las más importantes se sitúa en el ámbito de la política económica. Una de las contradicciones más obvias que se observa, es que los Estados han asumido la obligación de mejorar su posición competitiva en el mercado global modificando para ello las regulaciones de sus respectivos mercados de trabajo ${ }^{2}$ (Hall y Soskice, 2001) y comprometiéndose a reflejar en sus presupuestos el recorte del gasto social. Es decir, han aceptado los principios económicos neoliberales caracterizados por la desregulación, la reducción de los Estados de bienestar y la flexibilidad de los mercados de trabajo. Mientras, el discurso político declara como objetivos prioritarios la búsqueda de la cohesión social y la importancia de incrementar los niveles de igualdad entre los ciudadanos europeos. Estas transformaciones que ha impulsado el capitalismo global suponen también la erosión del pacto social en el que inicialmente estaba fundado el Estado Nacional (Petrella, 1996).

Durante este proceso, las fuerzas socialdemócratas trataron de construir una alternativa que situara el progreso social y económico al mismo nivel de interés y actuación política, aunque este objetivo nunca se logró. En cambio, la orientación hacia la austeridad presupuestaria y la limitación monetaria en Europa sí se ha puesto en práctica y ha generado tasas de crecimiento variables y altas tasas de desempleo (Christoph y Hofbauer, 2008).

El Modelo Social ha sido una seña de identidad europea. Se definió para ser un proyecto articulado en torno a la solidaridad colectiva, la equidad social y la eficiencia productiva, resultantes de los procesos de conflicto y cooperación en Europa en los tiempos modernos (Moreno y serrano, 2007:33). Sin embargo, en los últimos años se ha modificado debido a las presiones recibidas para facilitar la expansión del capital y avanzar en el "Workfare State" (Vis, 2007). Desde la Agenda de Lisboa de 2000 también han cambiado las referencias al Modelo, presentándolo como un argumento para pedir la reestructuración y restricción de los Estados de bienestar. El Modelo ha sido transformado en un factor productivo, que debe ser utilizado para tener éxito en los mercados globales (Christoph y Hofbauer, 2008) porque se considera que sus impactos sobre el crecimiento económico son positivos.

A pesar de hablar del Modelo Social Europeo, nos referimos a un proyecto que no es el resultado de un proceso de convergencia de las políticas sociales de los Estados. Antes bien, existen modelos diferentes en cada uno de ellos que Esping-Andersen (1990) clasificó en su tipología como: Nórdico, Continental, Anglosajón y Mediterráneo. Posteriormente, para aquilatar mejor los procesos internos a los que estos modelos han dado lugar, se han incluido en la clasificación variables como el género, que fue ignorado en la primera. Es decir, se incluye el papel de las mujeres en el Estado de bienestar y, particularmente, en los sistemas de cuidado.

En sus inicios, los Estados del bienestar estuvieron orientados a la protección frente a los Viejos Riegos Sociales, asegurando protección frente a los problemas derivados del desempleo, de la enfermedad y de la invalidez, y de la vejez. Pero desde hace décadas, las sociedades avanzadas se enfrentan a Nuevos Riesgos Sociales (Beck, 1998). Algunos de ellos son la extensión de la exclusión social; el rol que desempeñan

\footnotetext{
${ }^{2}$ Reforma laboral de 2010 aprobada por el gobierno del PSOE y la sucesiva en 2012, aprobada por el gobierno del PP.
} 
las mujeres que, lejos de favorecer su emancipación, refuerza la desigualdad de género; las consecuencias de recibir servicios y prestaciones de baja calidad a causa de la privatización de servicios públicos; y el envejecimiento demográfico.

La aparición de Nuevos Riesgos en el escenario social no significa que los Viejos hayan desaparecido, por ello los Estados de bienestar se enfrentan a un reto sin precedentes. A esta situación hay que añadir la existencia de un contexto aún más complejo que en décadas anteriores. De hecho, los países de la UE han abordado en distintos momentos reformas de sus Estados de bienestar. Ya en los noventa el gasto público de la UE-15 fue oscilando entre el recorte y el gasto. Algo más tarde, la puesta en marcha del mercado único, la necesidad de cambios en la economía europea y la reducción del déficit público se convierten en objetivos principales, y el discurso de la globalización se instrumentaliza para trata de modificar los pactos de solidaridad en los ámbitos internos de los Estados miembros (Moreno y Palier, 2005).

De hecho, la Agenda de Lisboa (2000) supuso un cambio en el sentido que tenía el Modelo Social cuando afirma la necesidad de una reestructuración para afrontar situaciones nuevas. Unas, derivan de la globalización, como la necesidad de competir en un mercado global y, otras, derivan de cambios macro sociales como por ejemplo, el envejecimiento demográfico. Las fórmulas para llevar a cabo tal cambio, según el discurso hegemónico, suponen pasar de un Modelo Social regulado a uno desregulado, basado en la flexibilidad de los mercados laborales, la activación de las políticas sociales y la privatización de los servicios públicos.

Actualmente, podríamos decir que Europa y su Modelo social se encuentran en una encrucijada histórica, dadas las fuerzas políticas y económicas que operan sobre él a través de dos escenarios: la existencia de otros modelos sociales y el modelo de construcción europea.

En cuanto al Modelo, Europa tendrá que decidir hacia cuál de ellos se dirige. Puede aproximarse al Modelo Neoesclavista Asiático (Moreno, 2013), cuya característica fundamental es que se apropia del valor económico de las personas aunque no garantiza su supervivencia (Bales, 2000) $)^{3}$. O puede parecerse al Modelo Remercantilizador americano, que defiende que el mercado es el mecanismo más eficiente para asignar recursos y bienestar. Estos dos modelos comparten el principio de que es necesario reducir los Estados del bienestar y el gasto público para competir en el ámbito global.

En cuanto al modelo de construcción de la Unión Europea los retos son enormes porque las políticas sociales siguen siendo competencia de cada Estado miembro, mientras que los retos y los riesgos cada vez tienen un componente más global. De hecho, en la Carta de Derechos Fundamentales de la Unión Europea (2000) ${ }^{4}$, los incluidos en ella no suponen una modificación de los umbrales de cada Estado, ni tampoco la puesta en marcha de un proceso que homogeneice éstos en toda la Unión, dado que se respetan expresamente las tradiciones constitucionales de cada miembro. En consecuencia, las políticas sociales y laborales son las únicas que pueden utilizarse para el ajuste interno en los Estados (Etxezarreta, 2008), según el Pacto Social Europeo.

\footnotetext{
${ }^{3}$ En nuestra realidad también existen grupos de población que "llevan a cabo cualquier tipo de trabajos a cambio de salarios de supervivencia" (Lucena, 2008: 127).

${ }^{4}$ La Carta revisada se proclamó en 2000 y la carta se hace vinculante para todos los países de la UE, excepto para Polonia y Reino Unido, con la firma del Tratado de Lisboa en diciembre de 2007.
} 
Los escenarios y procesos planteados inciden directamente sobre las condiciones de vida de los ciudadanos, ya que sus consecuencias son principalmente estructurales. De hecho, los cambios políticos, sociales y económicos relacionados con la globalización, junto con otros como el desarrollo tecnológico, han contribuido a un cambio social que, como decíamos, genera Nuevos Riesgos Sociales (Beck, 1998).

Después de décadas, se puede afirmar que el proceso de construcción europea no ha concluido con un Modelo Social único, pese a las directivas de la UE y de la adopción de unos mínimos comunes. Al contrario, observamos que, a pesar de los grandes consensos alcanzados para lograr los objetivos de igualdad y cohesión social, la construcción europea está suponiendo una erosión del Modelo, porque el pacto fundado en la solidaridad, en la ciudadanía y en la política social para hacer efectivos derechos sociales, se han transformado en un pacto basado en la competitividad, la activación y la fragmentación de las políticas sociales. Las consecuencias de estos procesos son la remercantilización de las relaciones sociales y el fortalecimiento de la familización (Espin-Andersen, 1990) en los sistemas de protección social.

En definitiva, la presión que sufren los Estados del bienestar procede de la concepción que tiene el modelo neoliberal del Estado del bienestar: la política social forma parte de una estructura que impide la competitividad con los países más pobres, por ello, el gasto social debe transformarse en un factor que permita la expansión del capital y que vincule (en mayor medida) los derechos laborales al empleo, de modo que se garantice la disciplina laboral (Etxezarreta, 2008). Esto significa, en la práctica, la desaparición de la universalidad de los sistemas de protección social. Por poner un ejemplo de este asunto tomaremos el sistema sanitario en España. Este sistema se considera un factor importante de desarrollo económico por ser un sector dinámico, que genera un número significativo de empleos y porque sus impactos sobre otros sistemas son muy relevantes. La aplicación del Real Decreto-ley 16/2012, de 20 de abril, de medidas urgentes para garantizar la sostenibilidad del Sistema Nacional de Salud y mejorar la calidad y seguridad de sus prestaciones, ha supuesto, sin embargo, el fin de la universalidad con la que, de hecho, funcionaba el sistema sanitario.

La influencia de la Unión sobre los sistemas nacionales se manifiesta, asimismo, en las normas promulgadas para la construcción del mercado interno y en las propias directivas (de transposición obligatoria al derecho nacional).

\section{La discapacidad en la normativa internacional y nacional}

Los procesos a los que nos hemos referido tienen su traducción en normas internacionales y nacionales que definirán cambios estructurales y cambios en las condiciones de vida de los individuos. En el ámbito de la discapacidad, la preocupación de las organizaciones internacionales y de los Estados es un hecho. La producción de normas como las que emanan de la Convención de la ONU sobre derechos de las personas con discapacidad, la Carta de derechos fundamentales de la Unión Europea y la Estrategia Europea sobre Discapacidad 2010-2020, entre otras, evidencian la preocupación y su disposición a implementar medidas para el logro de la igualdad y de la inclusión social.

La Convención de Naciones Unidas sobre derechos de personas con discapacidad (2006) tiene como propósito "promover, proteger y asegurar el goce pleno y en condiciones de igualdad de todos los derechos humanos y libertades fundamentales por todas las personas con discapacidad, y promover el respeto de su dignidad inherente" (artículo 1). 
Por su parte, La Estrategia Europea sobre Discapacidad 2010-2020, toma en consideración, además de los documentos citados arriba, el artículo 10 del Tratado de Funcionamiento de la UE, y declara que su objetivo es "capacitar a las personas con discapacidad para que puedan participar plenamente en la sociedad, en condiciones con el resto de la población". La estrategia también exhorta a los Estados miembros a colaborar con el fin de construir una Europa para todos, libre de barreras.

En España, se aprobó la Ley 39/2006, de 14 de Diciembre de Promoción de la autonomía personal y atención a las personas en situación de dependencia. Esta ley puso en marcha un sistema de atención a las necesidades de cuidado y promoción de la autonomía personal a través de una serie de recursos, ayudas y prestaciones a quienes los necesiten y al personal cuidador. Su implementación se ha producido de manera gradual y por niveles, de modo que los usuarios han sido incluidos en el sistema progresivamente, en función de su grado de dependencia.

Por su parte, la Ley 26/2011, de 1 de agosto, de adaptación normativa a la Convención Internacional sobre los Derechos de las Personas con Discapacidad (cuyo texto se consolida a través de la última modificación es el 31 de octubre de 2015), adapta los principios de la convención internacional a la normativa española.

En 2014 se aprueba el Plan de Acción de la Estrategia Española sobre Discapacidad. El eje del plan es eliminar las barreras que supongan algún tipo de discriminación contra las personas con discapacidad, mientras que el Plan anterior (2009-2012) perseguía la promoción de la autonomía personal reconociendo que la discapacidad es un elemento de la diversidad humana.

Estas Declaraciones, Tratados y Normas tienen un impacto notable sobre las personas dependientes o con discapacidades intelectuales, puesto que significan la concreción de principios en normas y en actuaciones que tienen un impacto directo sobre sus vidas.

Se podría pensar que, dada la energía y los recursos utilizados en poner en pie dichas normas y declaraciones, cabría una probabilidad alta de obtener una mejora de la calidad de vida de las personas con discapacidad intelectual, de las familias cuidadoras y que, en última instancia, se produciría una mejora sustantiva en términos de inclusión social. Sin embargo, se plantean algunas dudas a tenor de ciertos contenidos en estos documentos:

- La Ley 2011 de 21 de agosto, haciéndose eco de la Convención de ONU, anuncia un cambio de paradigma en las políticas sobre discapacidad, superando la perspectiva asistencial para abordar la perspectiva sobre derechos humanos. Lo cierto es que se trata de un avance importante, pero quedan excluidas situaciones como la garantía de los cuidados y de una calidad de vida digna. Cierto es que la discapacidad no significa necesariamente dependencia, más aún, si tenemos en cuenta la nueva definición que proporciona la Ley. Pone en relación la discapacidad con el ecosistema, resultando una situación negativa cuando esta relación no es adecuada. Sin embargo, la discapacidad es una realidad muy compleja de la que no puede quedar excluida la atención social mientras la igualdad legal no se haya traducido en igualdad real. Si esto ocurre, un alto porcentaje de personas con discapacidad se verán abocadas a tener una calidad de vida o unos cuidados deficientes que, por otra parte, irían contra la dignidad humana proclamada en la Convención. De hecho, las cuestiones que generan una vulnerabilidad mayor entre las personas con discapacidad intelectual es tener un acceso restringido a los cuidados y a la atención que necesitan. Estos recursos son 
caros y las necesidades son diversas, de modo que cuando no son de provisión pública, en muchos casos no es posible el acceso a ellos o su cantidad y calidad no son suficientes. El Estado, en nuestra opinión, debería recoger estos principios a través de su legislación.

- $\quad$ En España, se aprobó la Ley 39/2006, de 14 de Diciembre de Promoción de la autonomía personal y atención a las personas en situación de dependencia. Sin embargo, los servicios y recursos públicos que prevé para atender las necesidades de cuidado, no han sido implementados en su totalidad, y además, su dotación presupuestaria no se muestra suficiente para atender satisfactoriamente las situaciones sobre las que interviene.

- Otra debilidad de la producción normativa es el hecho de que no se mencione expresamente a las personas con discapacidad intelectual, dado que pueden ser consideradas las más vulnerables y las que necesitan más recursos asistenciales y económicos, en ocasiones para la mera subsistencia.

- $\quad$ En este caso particular, tanto las personas con discapacidad intelectual como sus cuidadores principales han visto reducidos sus derechos sociales en los últimos años, en lo que tiene que ver con la atención social (reducción de recursos y prestaciones), y en los derechos sociales de las personas cuidadoras (remuneración y cotizaciones a la seguridad social). Esta reducción de derechos y de recursos de atención, en nuestra opinión, entra en contradicción con todas las normativas y declaraciones internacionales.

- $\quad$ España se incluye en el ámbito de aplicación de la Estrategia Europea 2020 (aprobada en 2010) en la cual se aprueban unos objetivos cuantificados para hacer frente a la crisis económica y alcanzar un crecimiento sostenible, inteligente e integrador (Estrategia Española, p. 4). En nuestra opinión, esta estrategia tiene su traslación en España en el énfasis sobre la igualdad de oportunidades en el acceso y participación en el mercado de trabajo.

La crítica que planteamos aquí no es al desempeño del trabajo, sino a la orientación del Modelo Social Europeo que, de ser un elemento de protección social, se está transformando en un elemento destinado a mejorar la competitividad global. En este sentido se siguen las normas que rigen la globalización neoliberal, es decir, el modelo adopta un cariz orientado puramente al beneficio económico que obvia y minimiza los cuidados y la satisfacción de las necesidades básicas que no se consideran rentables. Y esta orientación incrementa el riesgo de exclusión de las personas con discapacidad intelectual y de sus cuidadores/as, puesto que las restricciones presupuestarias se traducen en una dotación menor de recursos, servicios y prestaciones. De hecho, uno de los objetivos de la Estrategia Española es la dinamización económica, en cuyo objetivo estratégico se puede leer: fomentar que los recursos de atención a la discapacidad constituyan un factor de dinamización económica, y sus objetivos operativos son: conectar la oferta y la demanda de productos y servicios accesibles; fomentar el vínculo entre calidad y accesibilidad universal en la gestión empresarial; apoyar a los sectores económicos con mayor potencial en la provisión de bienes y servicios accesibles. En definitiva, las personas con discapacidad se consideran como un grupo de consumidores potenciales cuyas necesidades se consideran una oportunidad económica para las empresas.

En suma, parece que no se despejan las incertidumbres sobre la atención a la discapacidad intelectual, porque, si bien es cierto que esta actividad normativa ha 
supuesto un cambio notable en el discurso sobre las personas con discapacidad, sobre su valoración y posición social y sobre la mejora de las condiciones de vida, es cierto también que cualquier avance es tortuoso, lento y susceptible de retrocesos rápidos como se ha observado estos años. Es necesario tener en cuenta en el diseño de las políticas sociales la perspectiva según la cual la eliminación de las barreras para garantizar la igualdad de oportunidades es una condición necesaria, pero en ningún caso es suficiente en el caso de las personas con discapacidad intelectual, porque requieren la satisfacción de necesidades de cuidado y de asistencia imprescindibles para el logro de una mínima inclusión social.

\section{Discusión y conclusiones}

Los desarrollos que se han abordado forman parte de un proceso de cambio social amplio que ha conducido a las sociedades desde la Modernidad a la Postmodernidad (Beck, 1998). Entre sus impactos encontramos algunos que han conducido a sociedades como la española a situaciones paradójicas. Por un lado, existe un discurso político que defiende los principios de universalidad y solidaridad de los sistemas de protección social. Y, por otro, existen unas condiciones acordadas y constitucionalizadas en relación a la política macroeconómica, que exigen el cumplimiento del déficit y de los compromisos incluidos en la Estrategia Europa 2020. Esta situación pone de manifiesto que debe reducirse el gasto social y remercantilizar (privatizar) determinadas prestaciones de servicios públicos, de modo que sean accesibles a través del mercado. La coincidencia de la orientación neoliberal del proceso de globalización y las reformas introducidas en el Modelo Social europeo, tienen como consecuencia lógica la reducción de las estructuras de los Estados del bienestar en los países, estructuras que dan cobertura a las personas que tienen más problemas para satisfacer sus necesidades a través del mercado. Los efectos de estos acuerdos, como decimos, son inmediatos. En el caso de las personas en situación de dependencia, entre las que se encuentra un buen número de personas con discapacidad intelectual, las consecuencias se concretan, principalmente, en reformas sucesivas de la Ley 39/2006 de Promoción de la autonomía personal y atención a las personas en situación de dependencia. Las reformas han reducido las prestaciones tanto a usuarios como a personal cuidador, han incrementado los copagos de los servicios y los centros, han dilatado la entrada en el sistema de los grados de discapacidad que estaban previstos en último lugar y han modificado los criterios de percepción de prestaciones. Es decir, los ha colocado en una situación de mayor vulnerabilidad social.

Por otra parte, la construcción del mercado interior de la Unión exige que el acceso al sistema sanitario no sea una barrera para la movilidad pero, al mismo tiempo, legislaciones como la española, a través del Real Decreto-ley 16/2012, de 20 de abril de medidas urgentes para garantizar la sostenibilidad del Sistema Nacional de Salud y mejorar la calidad y seguridad de sus prestaciones, modifica las condiciones de acceso al sistema de los propios nacionales y excluye a los inmigrantes en situación irregular.

Es posible que estas paradojas sean un reflejo propio de la globalización que, por un lado, genera un conjunto de problemas que solo pueden ser abordados globalmente, mientras que los Estados siguen siendo los actores políticos con legitimidad para abordarlos. En esta situación solo cabe desarrollar actuaciones altamente parciales cuyos resultados lo serán igualmente. 
Estas paradojas, en nuestra opinión, se producen precisamente porque las intenciones y declaraciones no avanzan hacia la definición de los derechos, la concreción de los mismos y la implementación de los medios y recursos que los hacen reales y efectivos. Es decir, existe una falta de adecuación entre fines y medios.

En definitiva, consideramos que es imprescindible trascender los niveles retórico, normativo (de análisis ético) y formal para reconocer y satisfacer en la práctica los derechos de ciudadanía de las personas con discapacidad intelectual de manera efectiva. Para alcanzar este objetivo es imprescindible proveer protección social suficiente y adecuada a cada tipo de necesidad, tanto de los individuos como de las personas que ejercen las tareas de cuidado, puesto que, si se carece de los medios, las personas con discapacidad intelectual serán las más expuestas a padecer una situación de exclusión social a lo largo de su vida.

\section{Referencias}

Bales, K. (2000). La nueva esclavitud en la economía global. Madrid: Siglo XXI.

Beck, U. (1998). La sociedad del riesgo: hacia una nueva modernidad. Barcelona: Paidós.

Beck, U. \& Beck-Gernsheim, E. (2003). La individualización. El individualismo institucionalizado y sus consecuencias sociales y políticas. Barcelona: Paidós.

Christoph, H. \& Hofbauer, I. (2008). El modelo social europeo entre la modernización competitiva y la resistencia frente al neoliberalismo. Revista de Economía Crítica (6), 15-26.

Del Pino, E. (2004). Debates sobre la reforma del Estado de Bienestar en Europa: conceptos, alcance y condiciones. Documento de Trabajo (4), 01-30.

Diario Oficial de la Unión Europea (2010). Carta de Derechos Fundamentales de la Unión Europea. http://www.boe.es/doue/2010/083/Z00389-00403.pdf [Acceso: 17.11.2016]

Esping-Andersen, G. (1990). The three worlds of welfare capitalism. Cambridge: Polity Press.

Etxezarreta, M. (2008). La evolución (perversa) de la política social de la Unión Europea. Revista internacional de filosofía política, 2008 (31), 123-138.

Giddens, A. (1990). The consequences of modernity. Cambridge: Polity Press.

Jónasdòttir, A. (1993). El poder del amor. ¿Le importa el sexo a la democracia? Madrid: Cátedra.

Lucena, H. (2008) 1854-2004: 150 años de la abolición de la esclavitud en Venezuela ¿presente y pasado de una misma realidad? Humania del Sur (4), 183-186. http://www.saber.ula.ve/dspace/handle/123456789/15443 [Acceso: 17.11.2016]

Meil, G. (2006). Padres e hijos en la España actual. Barcelona: Obra Social "La Caixa".

Ministerio de Sanidad y Política Social (2009-2012). III Plan de Acción para las Personas con Discapacidad. 
https://www.msssi.gob.es/ssi/discapacidad/docs/III_PAPCD.pdf

[Acceso: 17.11.2016]

Moreno, L. y Palier, B. (2005), "The Europeanization of Welfare: Paradigm shifts and social policy reforms" en Peter Taylor-Gooby (Ed.), Ideas and Welfare State Reform in Western Europe (145-175). Nueva York: Palgrave Macmillan.

Moreno, L. (2007). Europeización del Bienestar y activación. Politica y sociedad, 44(2), 31-46.

Moreno, L. (Ed.). (2009). Reformas de las políticas del bienestar en España. Madrid: Siglo XXI.

Moreno, L. (2013). La Europa Asocial. Madrid: Península. doi: https://doi.org/10.1093/europace/eut032

Petrella, R. (1996) "Globalization and Internationalization: the Dynamics of the Emerging World Order" en R. Boyer y D. Dache (Eds.), States Against market: the limits of Globalization (62-83) Londres: Routledge.

Real Decreto-ley 16/2012, de 20 de abril, de medidas urgentes para garantizar la sostenibilidad del Sistema Nacional de Salud y mejorar la calidad y seguridad de sus prestaciones. «BOE» núm. 98, de 24/04/2012

https://www.boe.es/boe/dias/2012/04/24/pdfs/BOE-A-2012-5403.pdf

[Acceso: 10.11.2016]

Simmel, G. (1990). The philosophy of money. London: Routledge.

Vis, B. (2007). States of welfare or states of workfare? Welfare state restructuring in 16 capitalist democracies, 1985-2002. Policy \& Politics, 35(1), 105-122. doi: https://doi.org/10.1332/030557307779657720

Soskice, D. W., \& Hall, P. A. (2001). Varieties of capitalism: The institutional foundations of comparative advantage. Oxford: Oxford University Press.

Fecha de recepción: 03/11/2017

Fecha de revisión: $24 / 11 / 2017$

Fecha de aceptación: 09/04/2018 